\title{
Voxel-wise glioblastoma-survival mapping: new tool, new questions
}

\author{
Philip de Witt Hamer ${ }^{1} \cdot$ Emmanuel Mandonnet ${ }^{2,3,4}$ (1)
}

Received: 28 March 2021 / Accepted: 30 March 2021 / Published online: 12 April 2021

(C) The Author(s), under exclusive licence to Springer-Verlag GmbH Austria, part of Springer Nature 2021, corrected publication 2021

Decisions on neurosurgical treatment in patients with presumed glioblastoma should be based on precise and accurate predictions of outcome. Patients expect from their neurosurgeon that advice will not be merely based on intuition or personal preference, but on realistic expectations towards oncological and functional outcome based on representative data from earlier patients in similar circumstances. Although extent of resection has been demonstrated to be a major prognostic factor [1], other patientrelated and tumor-related preoperative characteristics, including age, physical condition, and tumor volume have been repeatedly confirmed as relevant patient-related predictors [6]. After surgery, the molecular tumor profile, including IDH-1/2 and MGMT methylation status, and adjuvant treatment decisions on chemoradiotherapy and clinical trial participation become available, which also contribute substantially to life expectancy.

Fyllingen et al. have contributed an important next step towards understanding whether the tumor location holds prognostic information for survival [4]. A strong point is that their patient cohort is not a selection of the most complex glioblastomas, because the Norwegian healthcare system is not organized in tertiary referral centers for neuro-oncology. This renders their results more generalizable to patient populations from similar healthcare systems. They demonstrated tumors in central locations and the left anterior temporal lobe to coincide with shorter survival, and conversely tumors in superficial locations in the right dorsomedial temporal lobe and left superior frontal gyrus to coincide with longer survival. This builds upon findings by Ellingson et al. [3] in the use of tumor load maps to identify regions associated with shorter and longer survival.

Emmanuel Mandonnet

mandonnet@mac.com

1 Department of Neurosurgery, VU University Medical Center, Amsterdam, Netherlands

2 Frontlab, Institut du Cerveau (ICM), CNRS UMR 7225, INSERM U1127, Paris, France

3 Hôpital Lariboisière, Paris, France

4 Université de Paris, Paris, France
Nevertheless, this earlier work identified the left temporal regions to be associated with longer survival and right temporal regions with shorter survival. As likely explanation for this discrepancy, either analysis may be subject to false-positive findings. The fact that presence of tumor in the left temporal pole correlated with shorter survival when comparing survival groups $<6$ months with 6-24 months, but not when comparing $<6$ months and $>$ 24 months, may indicate that the findings should be interpreted as a methodological artefact. Moreover, tumor location was analyzed as single variable with survival as dependent categorical variable. In a first attempt to disentangle the effect on survival of voxel-wise tumor location from the effect of other known prognostic factors such as age or residual tumor volume, it could have been informative to perform the same voxel-wise analysis for patient subgroups characterized by age or residual tumor volume in addition to survival. If the obtained maps were not found to overlap with the ones found when grouping by survival, this would have been a strong argument to believe that the evidenced locations on figure 3 carry a true effect of better/worse survival, independently of other know prognosis factors.

Finally, Fyllingen et al. confirm that a glioblastoma involving the corpus callosum is associated with shorter survival [2]. Their shortest distance to the center of the third ventricle is a clever practical measure of deep-seated tumor locations, which they convincingly found to be negatively correlated with survival, partly depending on age. This distance is also closely related with the surgical strategy to biopsy [5], leaving the question open whether a tumor location closer to the center of the brain per se is the limiting factor for resection precluding survival benefit from surgical treatment, or whether this is a surrogate marker for an unfavorable phenotype that involves deeper brain locations more often, yielding poor survival outcome no matter the surgical strategy.

How does this new knowledge enable better decisions in our patients? The distance to the center of the brain may prove to be an independent prognostic factor to guide the decision whether to biopsy or to resect. As the authors state, this requires confirmation in other populations. To disentangle whether tumor location is independently related with shorter 
survival, methods should be developed to include tumor load map information in multivariable survival models.

\section{References}

1. Brown TJ, Brennan MC, Li M et al (2016) Association of the extent of resection with survival in glioblastoma: a systematic review and meta-analysis. JAMA Oncol 2(11):1460-1469

2. Chaichana KL, Jusue-Torres I, Lemos AM, Gokaslan A, CabreraAldana EE, Ashary A, Olivi A, Quinones-Hinojosa A (2014) The butterfly effect on glioblastoma: is volumetric extent of resection more effective than biopsy for these tumors? J Neuro-Oncol 120(3):625-634

3. Ellingson BM, Lai A, Harris RJ et al (2013) Probabilistic radiographic atlas of glioblastoma phenotypes. AJNR Am J Neuroradiol 34(3): $533-540$
4. Fyllingen EH, Bø LE, Reinertsen I, Jakola AS, Sagberg LM, Berntsen EM, Salvesen Ø, Solheim O (2021) Survival of glioblastoma in relation to tumor location: a statistical tumor atlas of a population-based cohort. Acta Neurochir. https://doi.org/10.1007/ s00701-021-04802-6

5. Müller DMJ, Robe PAJT, Eijgelaar RS et al (2019) Comparing glioblastoma surgery decisions between teams using brain maps of tumor locations, biopsies, and resections. JCO Clin Cancer Inform 3:1-12

6. Senders JT, Staples P, Mehrtash A, Cote DJ, Taphoorn MJB, Reardon DA, Gormley WB, Smith TR, Broekman ML, Arnaout O (2020) An online calculator for the prediction of survival in glioblastoma patients using classical statistics and machine learning. Neurosurgery 86(2):E184-E192

Publisher's note Springer Nature remains neutral with regard to jurisdictional claims in published maps and institutional affiliations. 